OPEN ACCESS

Edited by:

Robin Polt,

The University of Arizona

United States

Reviewed by: Yoshitoshi Kasuya,

Chiba University, Japan

Wladyslaw - Lason

Institute of Pharmacology of the

Polish Academy of Sciences, Poland

*Correspondence:

Hongguang BaO

baohongguang888@163.com

Specialty section:

This article was submitted to

Neuropharmacology,

a section of the journa

Frontiers in Neuroscience

Received: 17 December 2018 Accepted: 28 March 2019

Published: 24 April 2019

Citation:

Pan C, Si Y, Meng $Q$, Jing $L$, Chen L, Zhang $Y$ and Bao H (2019)

Suppression of the RAC1/MLK3/p38

Signaling Pathway by $\beta$-Elemene

Alleviates Sepsis-Associated

Encephalopathy in Mice.

Front. Neurosci. 13:358.

doi: 10.3389/fnins.2019.00358

\section{Suppression of the RAC1/MLK3/p38 Signaling Pathway by $\beta$-Elemene Alleviates Sepsis-Associated Encephalopathy in Mice}

\author{
Cailong Pan ${ }^{1}$, Yanna Si ${ }^{1}$, Qinghai Meng' ${ }^{2}$, Ling Jing ${ }^{1}$, Lu Chen ${ }^{3}$, Yong Zhang ${ }^{1}$ and \\ Hongguang Bao ${ }^{1 *}$
}

'Department of Anesthesiology, Nanjing First Hospital, Nanjing Medical University, Nanjing, China, ${ }^{2}$ School of Pharmacy, Nanjing University of Chinese Medicine, Nanjing, China, ${ }^{3}$ Jiangsu Key Laboratory of Neurodegeneration, Department of Pharmacology, Nanjing Medical University, Nanjing, China

It is still difficult to treat sepsis-associated encephalopathy (SAE) which is a diffuse brain dysfunction caused by sepsis, with excessive activation of microglia as one of the main mechanisms. Ras-related C3 botulinum toxin substrate 1 (RAC1) is proven to be a key molecule in the inflammatory signaling network. By using microglial cell line BV-2 and a mouse model of cecal ligation puncture (CLP), we herein evaluated the effects of $\beta$-elemene, an extract of Curcuma zedoaria Rosc., on RAC1 signaling in microglia. $\beta$-Elemene decreased the expressions of pro-inflammatory cytokines [tumor necrosis factor- $\alpha(\mathrm{TNF}-\alpha)$, interleukin-1 $\beta(\mathrm{IL}-1 \beta)$, and IL-6] and attenuated translocation of nuclear factor- $\mathrm{kB}(\mathrm{NF}-\kappa \mathrm{B})$ p65 from the cytosol to the nucleus in BV-2 cells after lipopolysaccharide (LPS) treatment. It also inhibited the activation of RAC1, mixedlineage protein kinase 3 (MLK3) and p38 mitogen-activated protein kinase (MAPK). The phosphorylation of the RAC1 Ser71 site was increased by $\beta$-elemene. Moreover, the learning and memory abilities of CLP mice in the water maze test and fear conditioning test were improved after $\beta$-elemene treatment. It reduced the expression of the microglial marker IBA1, significantly increased RAC1 Ser71 phosphorylation, and suppressed the RAC1/MLK3/p38 signaling activation and inflammatory response in the hippocampus. In conclusion, $\beta$-elemene effectively alleviated SAE in mice and inhibited the RAC1/MLK3/p38 signaling pathway in microglia, and might be an eligible potential candidate for SAE treatment.

Keywords: $\beta$-elemene, sepsis-associated encephalopathy, RAC1, MLK3, p38MAPK, microglia

\section{INTRODUCTION}

Sepsis-associated encephalopathy (SAE) is a diffuse brain dysfunction caused by sepsis. Sonneville et al. (2017) recently reported that 53\% of septic patients had SAE in intensive care unit (ICU), and SAE was associated with higher mortality rate, more consumption of ICU resources and longer hospital stay. In clinical practice, SAE is primarily manifested as changes of mental status, especially those of awareness/consciousness and cognition (Chaudhry and Duggal, 2014). Rodent models of sepsis suffer from long-term memory impairment (Barichello et al., 2005; Semmler et al., 2007; 
Chavan et al., 2012), and long-term potentiation deficiency has been found in the hippocampal sections prepared from mice receiving cecal ligation puncture (CLP) by electrophysiological techniques (Imamura et al., 2011). Besides, increase of $A \beta$ and synaptophysin in the hippocampus of CLP rats aggravates cognitive deficits (Schwalm et al., 2014). Collectively, the hippocampus plays important roles in sepsis-induced learning and memory deficits. However, the mechanism of SAE is still unclear, and the treatment outcomes remain unsatisfactory, thereby urgently requiring new therapies.

It is well-documented that neuroinflammation played a key role in the onset and progression of SAE (Semmler et al., 2008; Piva et al., 2015). Microglia mainly regulate inflammatory response in the brain, the excessive activation of which leads to central nervous system damage upon SAE (Michels et al., 2014). P38 mitogen-activated protein kinase (MAPK), as a member of the MAPK family, is involved in the production of inflammatory mediators and the regulation of cytoskeletal stability (Cuenda and Rousseau, 2007). By intraperitoneally injecting lipopolysaccharide (LPS) into C57 mice, Kelly et al. (2003) activated p38 and inhibited LTP in the hippocampus, which were reversed by a p38 inhibitor SB203580. P38 MAPK has also been reported to phosphorylate various transcription factors, triggering the upregulation of inflammatory genes in the case of SAE (Jacob et al., 2011). Additionally, mixed lineage kinase 3 (MLK3) is a MAPK kinase kinase (MKKK) that mediates the phosphorylation of p38 MAPK by binding ras-related C3 botulinum toxin substrate 1 (RAC1), a GTP-binding proteins (Gallo and Johnson, 2002). RAC1 dominantly participates in cellular physiological activities such as cell adhesion, proliferation and cytoskeleton regulation. Over-activated RAC1 is involved in pathological processes such as oxidative stress and inflammatory response upon sepsis (Sanlioglu et al., 2001). Additionally, RAC1 markedly affects microglial-mediated neuroinflammation and neurotoxicity (D’Ambrosi et al., 2014). After RAC1 changes from an inactive GDP-binding state to an active GTP-binding state, downstream signaling is activated. GTP binding of RAC1 is reduced through phosphorylation at Ser71 site (Kwon et al., 2000), which then decreases the binding of RAC1 to MLK3 and negatively regulates its downstream MAPK signaling pathway in the rat hippocampus, finally mitigating cerebral ischemic injury (Zhang et al., 2006). Therefore, enhancing RAC1 phosphorylation may suppress microglial activation and neuroinflammation, as a feasible SAE therapy.

Curcuma zedoaria Rosc. has been widely used as an antiinflammatory, analgesic or antitumor agent in clinical practice (Lobo et al., 2009). $\beta$-Elemene, an extract of Curcuma zedoaria Rosc., has well-documented broad-spectrum anticancer effects (Li et al., 2010). Yao et al. (2008) reported that $\beta$-elemene exerted antitumor effects on glioblastoma cells depending on p38 MAPK. Although $\beta$-elemene also has evident therapeutic effects on non-neoplastic diseases such as inflammation and atherosclerosis (Zhang et al., 2010; Liu et al., 2015), but the underlying mechanisms remain elusive.

We herein hypothesized that $\beta$-elemene relieved CLP-induced cognitive dysfunction by suppressing the RAC1/MLK3/p38 signal pathway to inhibit microglial activation. The findings provide new insights into the clinical effects of $\beta$-elemene on SAE and the mechanism.

\section{MATERIALS AND METHODS}

\section{Reagents}

$\beta$-Elemene (purity: 99\%) was purchased from Shanghai Gaolang Chemical Technology Co., Ltd. (Shanghai, China). Antibodies against IBA1, p-RAC1 (S71), RAC1, MLK3, p-MLK3 (T211 + S281), p65, p-p65 (S536), p38 and p-p38 (T180 + Y182) were bought from Abcam (CA, United States). Enhanced chemiluminescence reagents was obtained from PerkinElmer (Waltham, MA, United States). Secondary antibodies were provided by Chemicon (Temecula, CA, United States). PrimeScript ${ }^{\mathrm{TM}}$ II 1st Strand cDNA Synthesis Kit was purchased from Takara Biomedical Technology (Beijing) Co., Ltd. (Beijing, China). Antibody against glyceraldehyde-3phosphate dehydrogenase (GAPDH) and other reagents were bought from Sigma-Aldrich (St. Louis, MO, United States).

\section{CLP Mouse Model}

Male C57BL/6 mice aged 6-8 weeks were used to establish an SAE model by CLP. After anesthesia, the mice were ligated with a sterile 4-gauge wire to the distal end of the cecum (approximately $1.5 \mathrm{~cm}$ ). A sterile 22-gauge needle was used to puncture the center of the occluded cecum, that was pushed back into the abdominal cavity and sutured. For the sham group, the abdominal cavity was opened without ligation or perforation. Afterward, $1 \mathrm{ml}$ of prewarmed $\left(37^{\circ} \mathrm{C}\right)$ normal saline was intraperitoneally injected for rehydration. The mortality rate was $\leq 20 \%$. $\beta$-Elemene was dissolved in vehicle ( $0.1 \%$ DMSO) and the mice were intraperitoneally injected with different concentrations of $\beta$-elemene $(10,20,40 \mathrm{mg} / \mathrm{kg}$, once a day) for 7 days after CLP. $\beta$-Elemene was first injected $6 \mathrm{~h}$ after CLP.

\section{Sample Collection}

Two hours after the last injection of $\beta$-elemene the animals were sacrificed. Samples were collected and stored in two ways: (Sonneville et al., 2017) The intact brain of the mice was fixed in $4 \%$ paraformaldehyde at $4^{\circ} \mathrm{C}$ for over $24 \mathrm{~h}$. These samples were used for $\mathrm{HE}$ staining, immunohistochemical assay and immunofluorescence assay (Chaudhry and Duggal, 2014). The hippocampus tissue was frozen in liquid nitrogen for Western blot and RT-PCR.

\section{Morris Water Maze Test}

From the 7th day after surgery, Morris water maze training was performed in a $1.25 \mathrm{~m}$-diameter circular water pool that was filled with $25^{\circ} \mathrm{C}$ water to a depth of $30 \mathrm{~cm}$. A transparent $10 \mathrm{~cm}$ diameter columnar escape platform was placed $1 \mathrm{~cm}$ below the water surface. The time that a mouse entered water to climb the platform was defined as the latency time. If the mouse did not find the platform within $60 \mathrm{~s}$, it was guided to the platform, placed there for $20 \mathrm{~s}$ and then removed. During training, the platform was placed in a quadrant of the pool, and the mouse was started from one of the 4 quadrants, facing the wall. The 
experiments were conducted at the same time every day for four consecutive days. Subsequently, the number and time of a mouse entering the target quadrant within $60 \mathrm{~s}$ were recorded (Liu et al., 2018).

\section{Fear Conditioning Test}

From the 7 th day after surgery, fear conditioning test was conducted by placing the mice in a $70 \%$ ethanol-wiped test box for conditioned reflex training by sound stimulation and foot electrical stimulation. The mice were placed in a chamber with an energizable bottom. After 2 min of adaption, a singlefrequency sound signal $(4.5 \mathrm{kHZ}, 60 \mathrm{~dB}, 30 \mathrm{~s})$ was given to the mice for 4 times (interval: $3 \mathrm{~min}$ ). Then an electric shock ( $1 \mathrm{~mA}, 5 \mathrm{~s}$ ) and an audible signal as the prompt were given 6 times (interval: $3 \mathrm{~min} ; 18 \mathrm{~min}$ in total), and simultaneously ended. After $24 \mathrm{~h}$, a context test to evaluate hippocampusdependent memory was performed by placing the mice in the same chamber again without any stimulation. Cognitive deficits was assessed by measuring the amount of time the mouse demonstrated "freezing behavior," which is defined as a completely immobile posture except for respiratory efforts (Wiltgen et al., 2005).

\section{Hematoxylin-Eosin (HE) Staining}

The samples fixed in paraformaldehyde were dehydrated with $70,80,90,95$, and $100 \%$ ethanol solutions after being washed with flow water, transparentized with xylene, and paraffinembedded into tissue blocks. The blocks were thereafter cut into $4 \mu \mathrm{m}$-thick sections, deparaffinized in xylene, rehydrated in $100,95,80$, and $75 \%$ ethanol solutions, and rinsed with water for $5 \mathrm{~min}$. Then the sections were stained with hematoxylin for $4 \mathrm{~min}$, rinsed with water for $15 \mathrm{~min}$, differentiated with $1 \%$ hydrochloric acid-ethanol solution for $5 \sim 30 \mathrm{~s}$ until they became red, and rinsed with water for about $15 \mathrm{~min}$. After being stained with eosin for $90 \mathrm{~s}$, the sections were dehydrated with ethanol solutions at ascending concentrations, transparentized with xylene, covered with clean coverslips by using 1-2 drops of neutral resin, and quickly observed under a microscope.

\section{Western Blotting}

Tissues or cells were placed in a pre-cooled glass grinder, added pre-cooled lysis buffer containing PMSF and phosphatase inhibitors, and thoroughly ground for lysis. After addition of an appropriate amount of $5 \times$ loading buffer, proteins were separated by SDS-PAGE and transferred to PVDF membranes. The membranes were blocked with $5 \%$ bovine serum albumin (BSA) at room temperature for $1 \mathrm{~h}$ and then incubated with specific primary antibodies against GAPDH(1:5000), IBA1 (1:1000), p-RAC1 (S71) (1:1000), RAC1 (1:1000), MLK3 (1:5000), p-MLK3 (T211 + S281) (1:1000), p65(1:1000), p-p65 (S536) (1:1000), p38(1:1000) and p-p38 (T180 + Y182) (1:1000) overnight at $4^{\circ} \mathrm{C}$. The membranes were then developed by enhanced chemiluminescence reagents with secondary antibodies. The data were analyzed with the Molecular Imager (Gel DocTM XR, 170-8170, United States) and the associated software Quantity One-4.6.5 software (Bio-Rad Laboratories, United States).

\section{Immunohistochemical Staining}

The tissue was taken out, and fixed in a paraformaldehyde solution and prepared into paraffin blocks. The blocks thereafter cut into $4 \mu \mathrm{m}$-thick sections, deparaffinized and rehydrated. Endogenous peroxidase in the tissue sections was inactivated with $3 \% \mathrm{H}_{2} \mathrm{O}_{2}$. After being blocked by goat serum, the sections were incubated with primary antibodies overnight at $4^{\circ} \mathrm{C}$ and then with secondary antibodies for $1 \mathrm{~h}$ at $37^{\circ} \mathrm{C}$, stained with $\mathrm{DAB}$ color development solution, counterstained with hematoxylin for $4 \mathrm{~min}$, and observed under the microscope.

\section{RAC1 Assay}

Rac activation assays were performed according to the manufacturer's protocol of RAC1 pull-down activation assay biochem kit (Cytoskeleton, United States).

\section{RT-PCR}

Total RNA was extracted by using Trizol reagent according to the manufacturer's instructions and reverse-transcribed into cDNA. PCR experiments were then conducted with ABI7500 system (Life Technologies, United States) by TB Green ${ }^{\text {TM }}$ Premix Ex $\mathrm{Taq}^{\mathrm{TM}}$ (Tli RNaseH Plus) and ROX plus kit [Takara Biomedical Technology (Beijing) Co., Ltd., China]. GAPDH was used as a housekeeping gene. The mRNA levels were calculated by using the ${ }^{\Delta \Delta}$ CT method. The sense and antisense primers (TNF- $\alpha$, IL-1 $\beta$, IL-6, and GAPDH) as follows were purchased from Sangon Biotech (Shanghai) Co., Ltd. (China). TNF- $\alpha$ : $5^{\prime}$ TACTGAACTTCGGGGTGATTGGTCC- $3^{\prime}$ and $5^{\prime}$-CAGCCTT GTCCCTTGAAGAGAAC-3'. IL-1 $\beta$ : 5'-GCACTACAGGCTCC GAGATGAAC- $3^{\prime}$ and 5'-TTGTCGTTGCTTGGTTCTCCTTG T-3'. IL-6: 5' -CCGGAGAGGAGACTTCACAG-3' and 5'-GGA AATTGGGGTAGGAAGGA-3'. GAPDH: $5^{\prime}$-ACCACAGTCCAT GCCATCAC- $3^{\prime}$ and 5'-CACCACCCTGTTGCTGTAGCC-3'.

\section{Cell Culture}

BV-2 cells were cultured in DMEM containing 10\% fetal bovine serum, $100 \mathrm{U} / \mathrm{mL}$ penicillin, and $100 \mathrm{mg} / \mathrm{mL}$ streptomycin in a $37^{\circ} \mathrm{C}$ incubator containing $5 \% \mathrm{CO}_{2}$. $\beta$-Elemene was dissolved in $0.1 \%$ DMSO, and the cells were treated with different concentrations of $\beta$-elemene $(1,5,25 \mu \mathrm{M})$ for $24 \mathrm{~h}$.

\section{Nuclear Factor-кB (NF-кB) P65 Nuclear Translocation Assay}

BV-2 cells were plated in culture dishes with chamber slides inside, treated with LPS $(1 \mu \mathrm{g} / \mathrm{ml})$ for $12 \mathrm{~h}$ with or without $\beta$-elemene $(25 \mu \mathrm{M})$, fixed by $4 \%$ paraformaldehyde for $30 \mathrm{~min}$, blocked with $1 \%$ BSA, and incubated with primary antibodies overnight at $4^{\circ} \mathrm{C}$ and with secondary antibodies for $1 \mathrm{~h}$ at room temperature. After being stained with $1 \mu \mathrm{g} / \mathrm{mL}$ DAPI for $1 \mathrm{~min}$, the cells were observed and photographed using a fluorescence microscope. 


\section{Statistical Analysis}

Statistical analysis was carried out using ANOVA, with Bonferroni posttest as the post hoc test. The results were expressed as mean \pm standard deviation. $P<0.05$ was considered statistically significant. All analyses were performed with GraphPad Prism Version 5.01 (GraphPad Software Inc., San Diego, CA, United States).

\section{RESULTS}

\section{$\beta$-Elemene Suppressed LPS-Induced Inflammation in BV-2 Cells}

To investigate the effect of $\beta$-elemene on LPS-induced microglial activation in vitro, we used a mouse microglial cell line BV2 , an alternative model system for primary microglia cultures or animal experiments examining brain inflammation (Henn et al., 2009). LPS treatment significantly upregulated the mRNA expressions of pro-inflammatory cytokines TNF- $\alpha$, IL$1 \beta$, and IL-6, which were significantly reduced by $\beta$-elemene in a dose-dependent manner (Figure 1A). Hence, $25 \mu \mathrm{M}$ of $\beta$-elemene was selected for subsequent experiments in vitro. Besides, $\beta$-elemene alone $(25 \mu \mathrm{M})$ hardly affected the mRNA expressions of pro-inflammatory cytokines. In addition, LPS treatment significantly facilitated the translocation of p 65 NF- $\mathrm{B}$ from the cytoplasm to the nucleus, which was attenuated by $\beta$-elemene (Figure 1B).

\section{$\beta$-Elemene Inhibited the Activation of RAC1, MLK3, and p38 in BV-2 Cells}

Then we tested the activation of the RAC1/MLK3/p38 signaling pathway in BV-2 cells. The GTP-RAC1 was significantly increased by LPS induction, which was attenuated by $\beta$-elemene treatment (Figure 2A). The phosphorylation of MLK3 in BV-2 cells was significantly stronger than that of the control group, which was also weakened by $\beta$-elemene treatment (Figure 2A). Moreover, Western blotting exhibited that $\beta$-elemene significantly reduced the phosphorylation of p38 (Figure 2B). Interestingly, it significantly enhanced the phosphorylation of RAC1 Ser71 (Figure 2A). Taken together, the phosphorylated RAC1 Ser71 site was is involved in the regulation of RAC1 activity by $\beta$-elemene.

\section{$\beta$-Elemene Improved Learning and Memory in Septic Mice Induced by CLP}

We then evaluated the effects of $\beta$-elemene on the learning and memory of SAE mice. The water maze training showed that latency time was significantly prolonged after CLP compared to that of the sham group (Figure 3A), which was significantly shortened by $\beta$-elemene dose-dependently. There was no significant difference in the latency time between the $\beta$-elemene
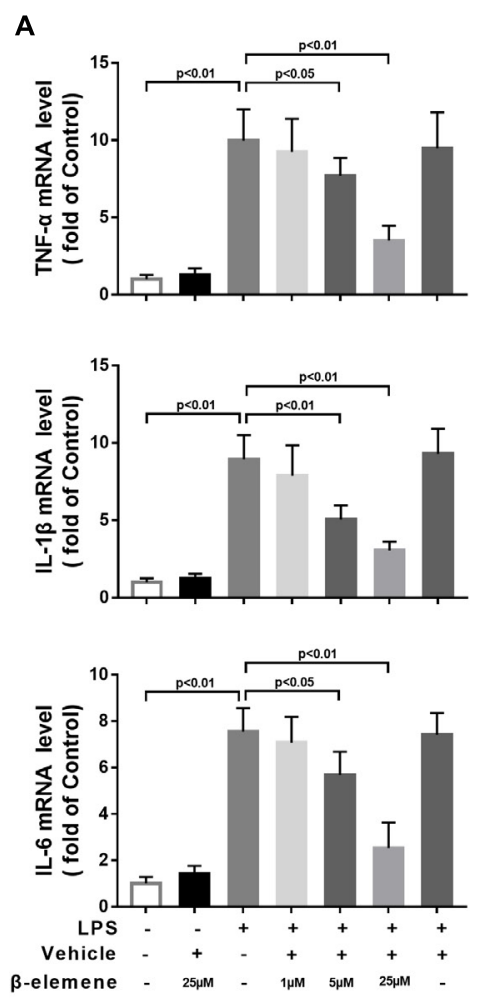
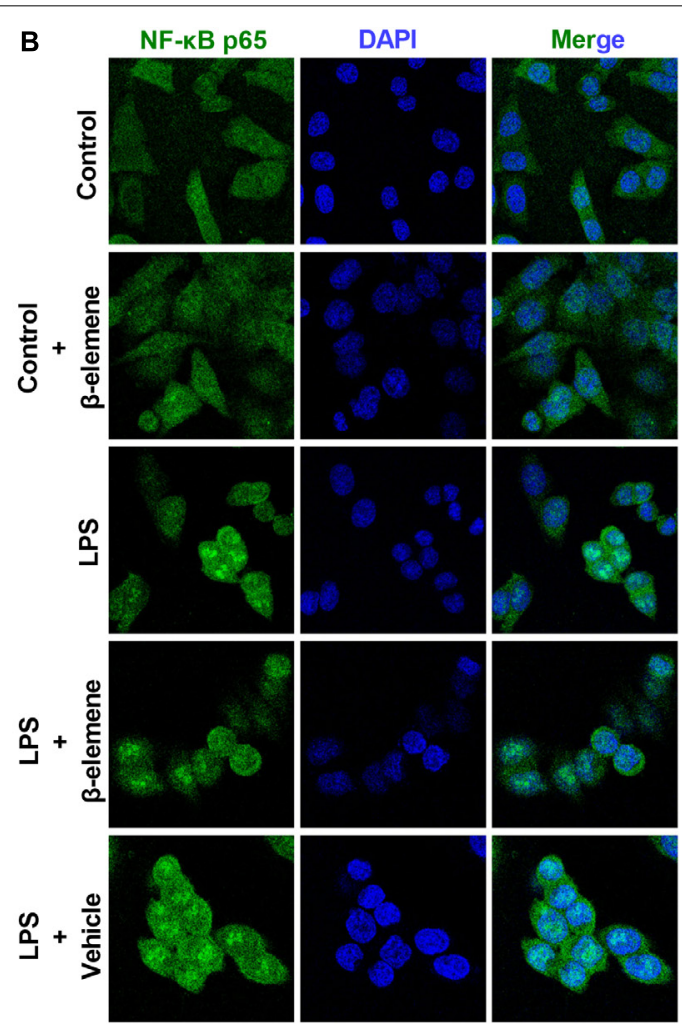

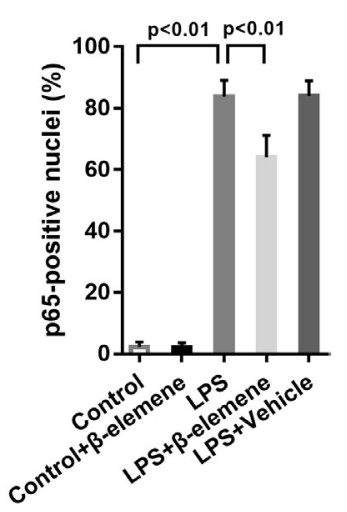

FIGURE 1 | $\beta$-Elemene suppressed LPS-induced inflammation in BV-2 cells. (A) $\beta$-Elemene suppressed LPS-induced upregulation of mRNA expressions of TNF- $\alpha$ $[F(6,21)=23], I L-1 \beta[F(6,21=26.4)]$, and IL-6 [F(6, $21=27.68)]$. GAPDH was used as a loading control. (B) $\beta$-Elemene reduced the NF- $\mathrm{BB}$ translocation from the cytosol to the nucleus. $\mathrm{F}(4,15)=244.6 . n=4$. Two-way or one-way ANOVA revealed a significant difference at $p<0.05$. 

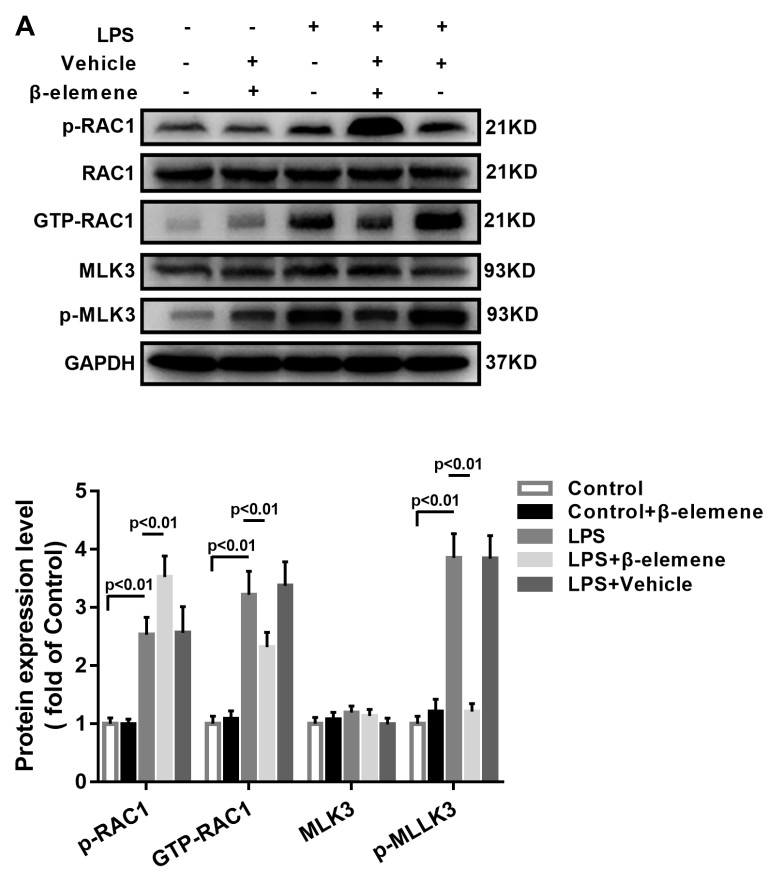
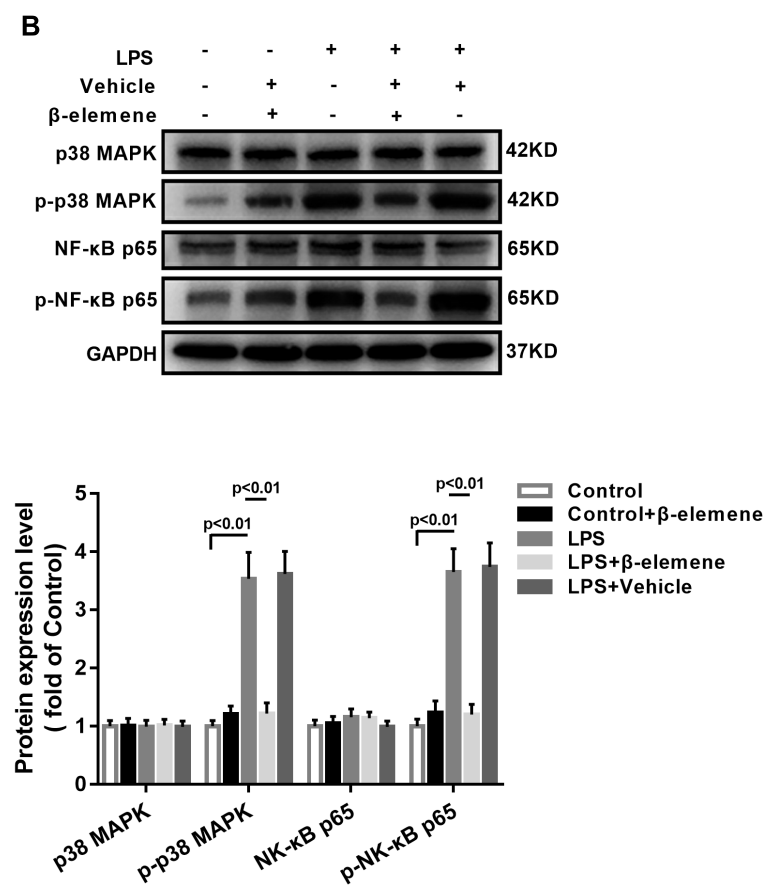

FIGURE 2 | $\beta$-Elemene enhanced phosphorylation of RAC1 and weakened activation of RAC1, MLK3, and p38 in BV-2 cells. (A) $\beta$-Elemene enhanced RAC1 phosphorylation $[F(4,15)=42.7]$ and weakened GTP-RAC1 production $[F(4,15)=40.61]$ and MLK3 phosphorylation $[F(4,15)=79.78]$. Phosphorylation of RAC1 was normalized to that of RAC1. Other protein expressions were normalized to that of GAPDH. (B) $\beta$-Elemene significantly reduced the phosphorylation of p38 $[\mathrm{F}(4$, $15)=63.23]$ and p65 $[F(4,15)=69.23]$ normalized to that of GAPDH. $n=4$. Two-way ANOVA revealed a significant difference at $p<0.05$.

group and the sham group, or between the CLP group and the CLP + vehicle group (Figure 3B). After training, the platform was removed, and the time spent in the target quadrant and the number of platform crossings were detected. The time spent by CLP mice in the target quadrant was significantly shorter than that of the sham group, which, however, was significantly extended by $\beta$-elemene (Figure 3C). Moreover, the number of CLP mice crossing the platform was significantly reduced compared to that of the sham group, whereas $\beta$-elemene significantly increased the number in a dose-dependent manner (Figure 3D). Additionally, the fear conditioning test showed that the freezing time of the CLP group mice was significantly shorter than that of the sham group, which was prolonged by $\beta$-elemene treatment (Figure $3 \mathrm{E}$ ). Hence, $40 \mathrm{mg} / \mathrm{kg}$ of $\beta$-elemene was selected for subsequent experiments. Notably, $\beta$-elemene administration did not affect the learning or memory of the sham group, and the placebo effect of vehicle on CLP mice was negligible.

\section{$\beta$-Elemene Reduced Microglial Marker IBA1 Expression in the Hippocampus of CLP Mice}

We further examined the hippocampal changes of CLP mice. HE staining showed that no abnormalities in brain cells were observed in the sham group. In contrast, largest amount of nuclear pyknosis were observed in the CLP groups. The dentate gyrus region changed most significantly. Moreover, mice given $\beta$-elemene showed significantly fewer abnormal cells compared to the CLP group (Figure 4A). Furthermore, Western blotting and immunofluorescence assay showed that the hippocampal expression of microglial marker IBA1 was significantly raised in CLP mice compared with that of the sham group, which was reduced by $\beta$-elemene treatment (Figures $4 B, 5$ ).

\section{$\beta$-Elemene Increased RAC1 Phosphorylation and Suppressed Its Activation in the Hippocampus}

Immunohistochemical assay exhibited that CLP itself induced moderate phosphorylation of RAC1, whereas $\beta$-elemene administration significantly augmented RAC1 Ser71 phosphorylation (Figure 6A). Western blotting results further confirmed that $\beta$-elemene significantly increased RAC1 phosphorylation and decreased GTP-RAC1 in the mouse hippocampus (Figure 6B).

\section{$\beta$-Elemene Reduced MLK3 Phosphorylation in the Hippocampus}

Immunohistochemical assay showed that the phosphorylation of MLK3 in the hippocampus of CLP mice was significantly enhanced compared with that of the sham group, which was attenuated by $\beta$-elemene treatment (Figure 7A). In addition, $\beta$-elemene significantly weakened the phosphorylation of MLK3 in the hippocampus, as suggested by Western blotting (Figure 7B). 
A

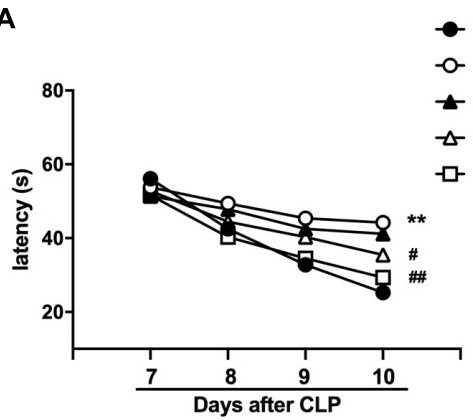

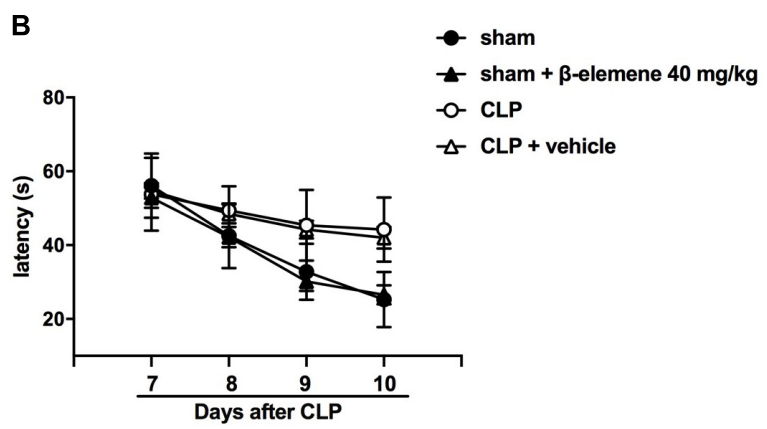

B

O- CLP

- CLP + $\beta$-elemene $10 \mathrm{mg} / \mathrm{kg}$

$\checkmark \mathrm{CLP}+\beta$-elemene $20 \mathrm{mg} / \mathrm{kg}$

CLP $+\beta$-elemene $40 \mathrm{mg} / \mathrm{kg}$
C

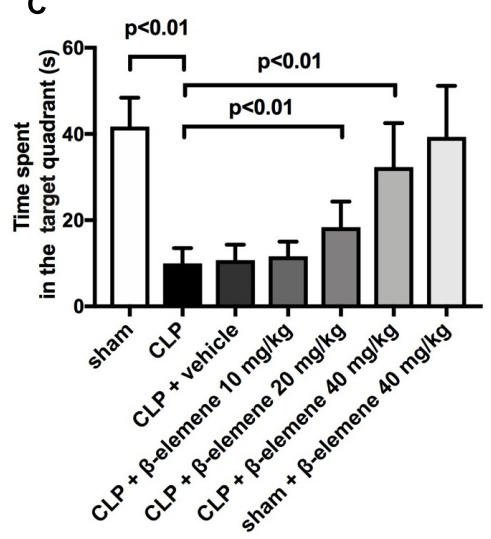

D

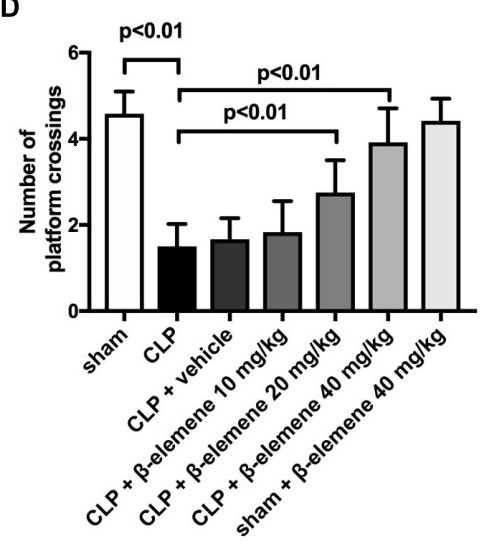

$\mathbf{E}$

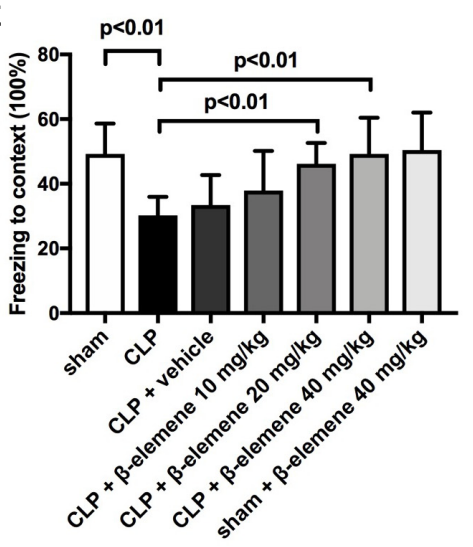

FIGURE 3 | $\beta$-Elemene improved learning and memory in septic mice induced by CLP. (A) $\beta$-Elemene significantly shortened CLP-induced prolongation of CLP-induced latency time. $F(6,308)=11$. (B) $\beta$-Elemene did not affect the latency time of the sham group and vehicle did not influence that of CLP mice. $F(6,308)=11$. (C) $\beta$-Elemene extended the time spent in the target quadrant. $F(6,77)=45.56$. (D) $\beta$-Elemene significantly increased the number of platform crossings. $F(6,77)=54.78$. (E) $\beta$-Elemene prolonged the freezing time in the context test. $F(6,77)=8.943 . n=12$. Two-way or one-way ANOVA revealed a significant difference at ${ }^{*} p<0.05$ and ${ }^{* *} p<0.01$ versus sham group; and ${ }^{\#} p<0.05$ and ${ }^{\# \#} p<0.01$ versus CLP group.

\section{$\beta$-Elemene Reduced p38 MAPK Phosphorylation, and Expressions of Pro-inflammatory Cytokines in the Hippocampus of CLP Mice}

We further assessed the effect of $\beta$-elemene on CLP-induced upregulation of p38 MAPK and NF- $\mathrm{B}$ p65 phosphorylation. Western blotting presented that $\beta$-elemene significantly reduced the phosphorylations of both p38 MAPK and NF- $\kappa$ B 65 (Figure 8A). Furthermore, RT-PCR indicated that CLP mice had higher mRNA expression levels of pro-inflammatory molecules (TNF- $\alpha$, IL-1 $\beta$, and IL-6) than those of the sham group, whereas $\beta$-elemene effectively reduced them (Figure 8B).

\section{DISCUSSION}

Herein, $\beta$-elemene suppressed the activation of RAC1, MLK3, p38 MAPK, and NF-kB p65, as well as significantly decreased the expressions of pro-inflammatory cytokines in BV-2 cells and the hippocampus of mice. Moreover, it mitigated the learning/memory deficit in septic mice induced by CLP. Compared with the CLP group, $\beta$-elemene lowered the expression of the microglial marker IBA1 and increased the phosphorylation of RAC1 in the hippocampus. Taken together, $\beta$-elemene may be able to combat SAE via the RAC1/MLK3/p38 signaling pathway, as an attractive treatment candidate.

Accumulating evidence has proven that RAC1, as a key molecule in the inflammatory signaling network, controlled the progression of inflammation. Kuijk et al. (2008) found that RAC1 was responsible for the release of pro-inflammatory cytokines, which, when inhibited, significantly decreased IL-1 $\beta$. Zhang et al. (2011) reported that RAC1 was activated during LPS stimulation, thereby inducing TNF- $\alpha$ expression. Furthermore, Yao et al. (2011) used RAC1 inhibitor NSC23766 to significantly attenuate pro-inflammatory cytokines release, inflammatory cell infiltration, neutrophil migration and endothelial cell permeability in a mouse model of LPS-induced pulmonary inflammation. Similarly, Hwaiz et al. (2013) found that RAC1 was an effective target for protecting against lung injury during sepsis. NSC23766 markedly reduced CLP-triggered neutrophil infiltration, edema formation, and lung damage. Qin et al. (2015) also thought that RAC1 may be an effective therapeutic target for inflammation and sepsis. In this study, we found that inhibiting RAC1 activity also had protective effects on SAE. Meanwhile, the phosphorylation of RAC1 Ser71 site may be 


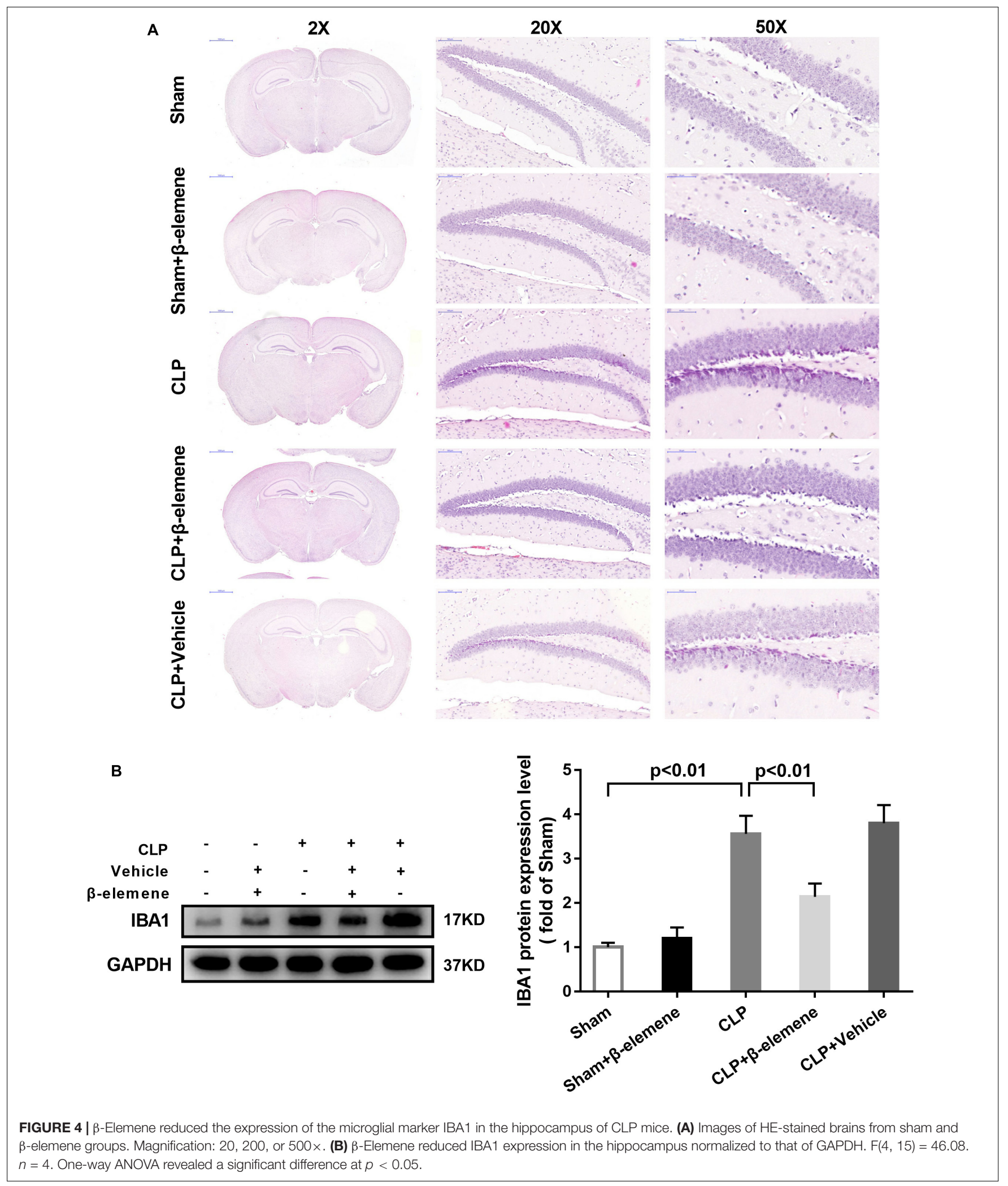

involved in the reduction of GTP-RAC1 by $\beta$-elemene. Although RAC1 Ser-71-phosphorylation weakened GTP binding without affecting the GTPase activity, phosphorylation predominantly modulated the subcellular localization of the GTPase, thereby negatively affecting its activity (Kwon et al., 2000; Riento et al., 2005; Wiltgen et al., 2005; Zhang et al., 2006, 2010, 2011; 

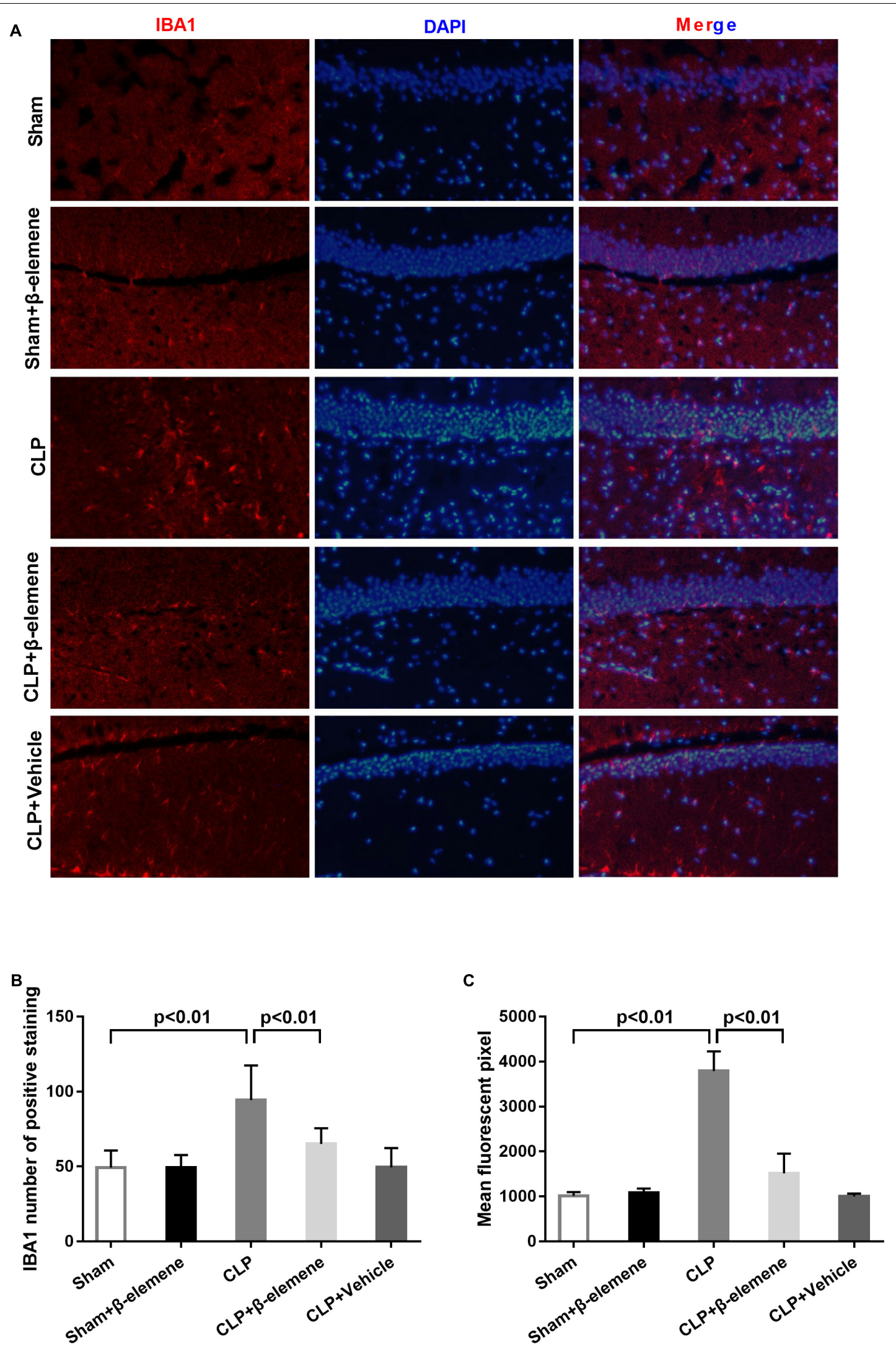

FIGURE 5 | Continued 
FIGURE 5 | $\beta$-Elemene reduced microglial activation in the hippocampus of CLP mice. (A) Immunofluorescence staining of IBA1 in the brains of different groups. Magnification: 200x. (B) IBA1 numbers of positively stained hippocampal dentate gyri in different groups. $F(4,15)=53.87$. (C) Mean fluorescent pixels of IBA1 in the hippocampal dentate gyri of different groups. $F(4,15)=8.416 . n=4$. One-way ANOVA revealed a significant difference at $p<0.05$.
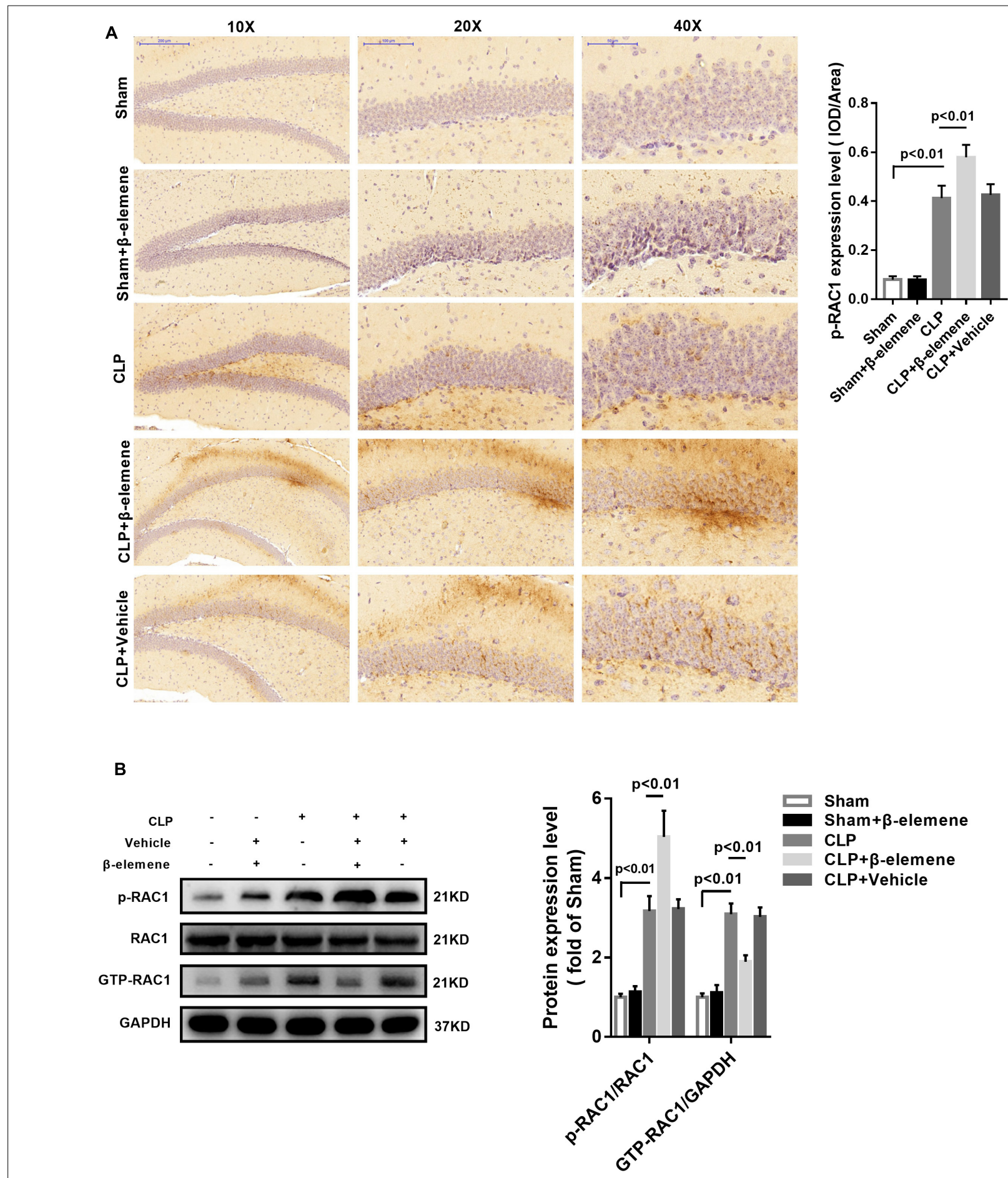

FIGURE 6 | $\beta$-Elemene increased the RAC1 phosphorylation in the mouse hippocampus. (A) Immunohistochemical staining showed that $\beta$-elemene significantly facilitated RAC1 phosphorylation. $F(4,15)=102$. (B) Western blotting exhibited that $\beta$-elemene significantly increased RAC1 phosphorylation $[F(4,15)=72.76]$ and decreased GTP-RAC1 $[F(4,15)=63.42]$ detected by western blotting in the hippocampus. Phosphorylation of RAC1 was normalized to that of RAC1. GTP-RAC1 expression was normalized to that of GAPDH. $n=4$. One-way ANOVA revealed a significant difference at $p<0.05$. 


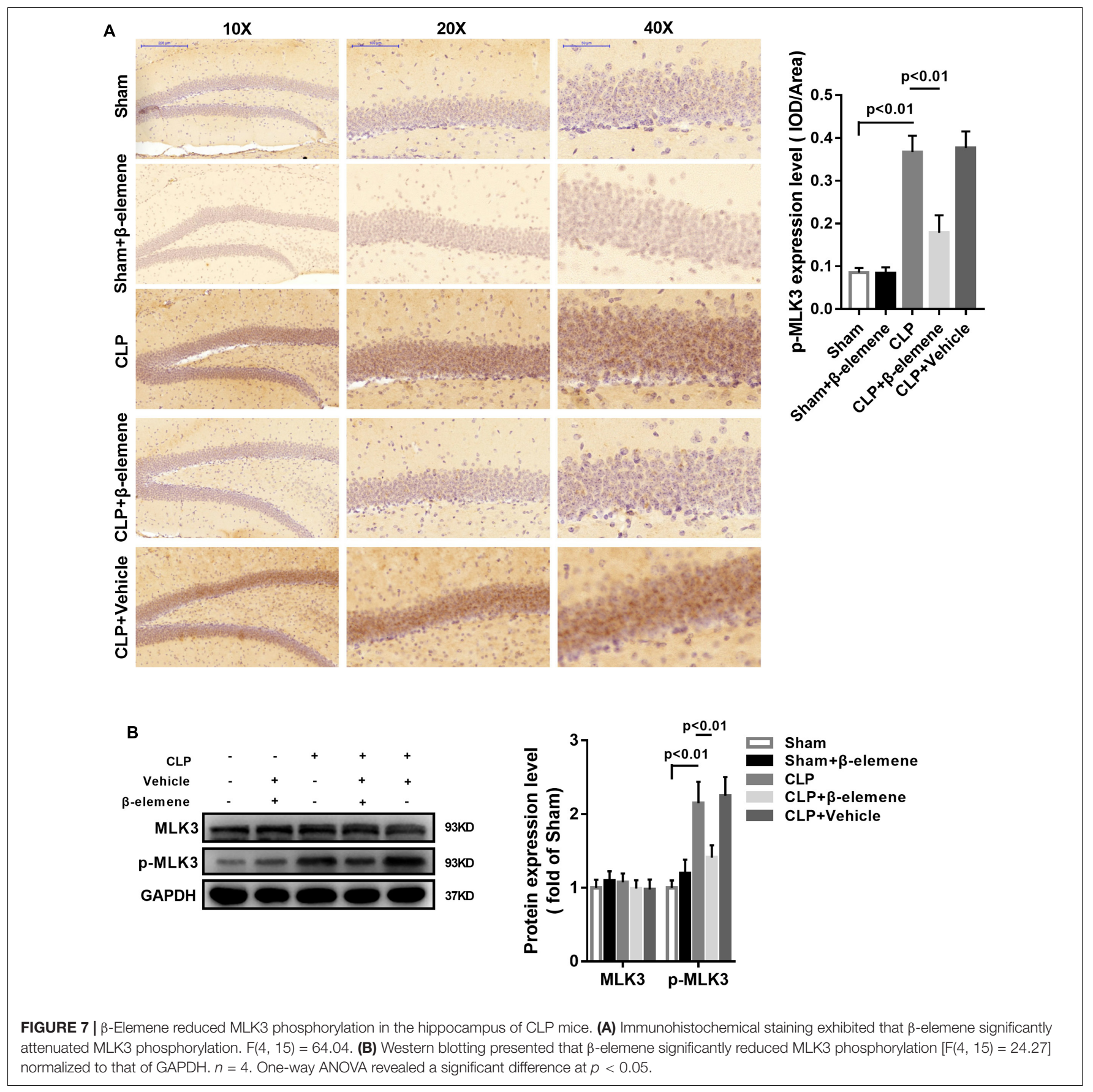

Kuijk et al., 2008; Yao et al., 2008, 2011; Henn et al., 2009; Lobo et al., 2009; Schoentaube et al., 2009; Li et al., 2010; Schwarz et al., 2012; Hwaiz et al., 2013; Liu et al., 2015, 2018; Qin et al., 2015). In addition, RAC1 Ser71 was moderately phosphorylated in the hippocampus of CLP mice (Figures 6A,B). RAC1 Ser71 phosphorylation in the CLP mice may be implicated as a subsequent protective reaction by the cell itself, but not a damage factor in the pathological process of SAE. In this study, for the first time, we found that $\beta$-elemene suppressed the inflammatory responses in CLP mice and LPS-exposed BV-2 cells likely by augmenting RAC1 Ser71 phosphorylation.
RAC1 has been reported to play important roles in cell movement, tumor angiogenesis and invasion/metastasis (Bid et al., 2013). Since RAC1 inhibitor NSC23766 is capable of combating breast cancer and lung cancer (Zuo et al., 2006; Hernandez et al., 2010; Yoshida et al., 2010; Chen et al., 2011), the antitumoral effects of $\beta$-elemene may also involve the same pathway. In addition, $\beta$-elemene can potently inhibit pro-inflammatory cytokines and protect against endotoxin-induced inflammation (Fang et al., 2018). Similarly, we found that $\beta$-elemene reduced the expressions of pro-inflammatory factors in the mouse model of SAE 

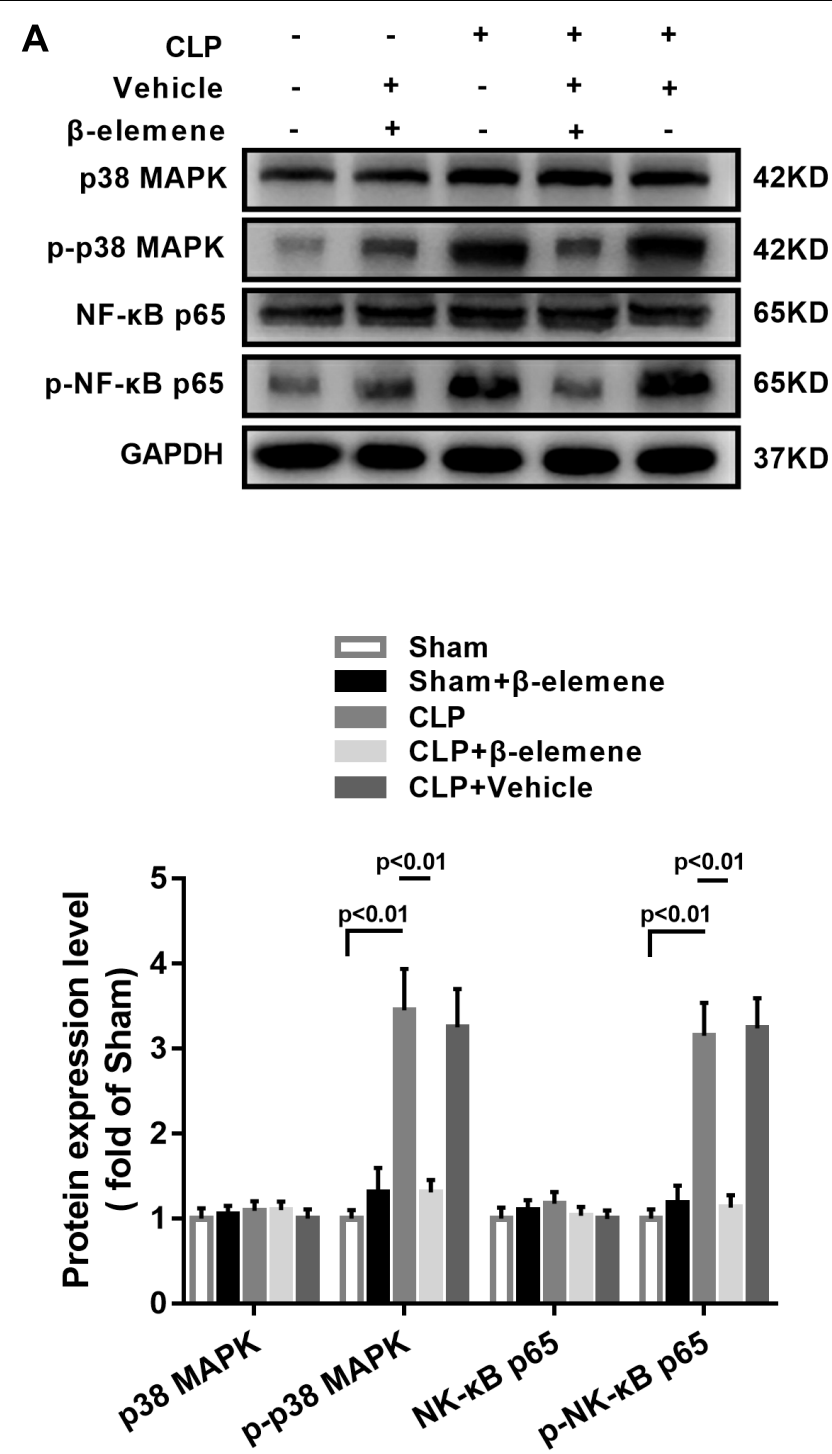
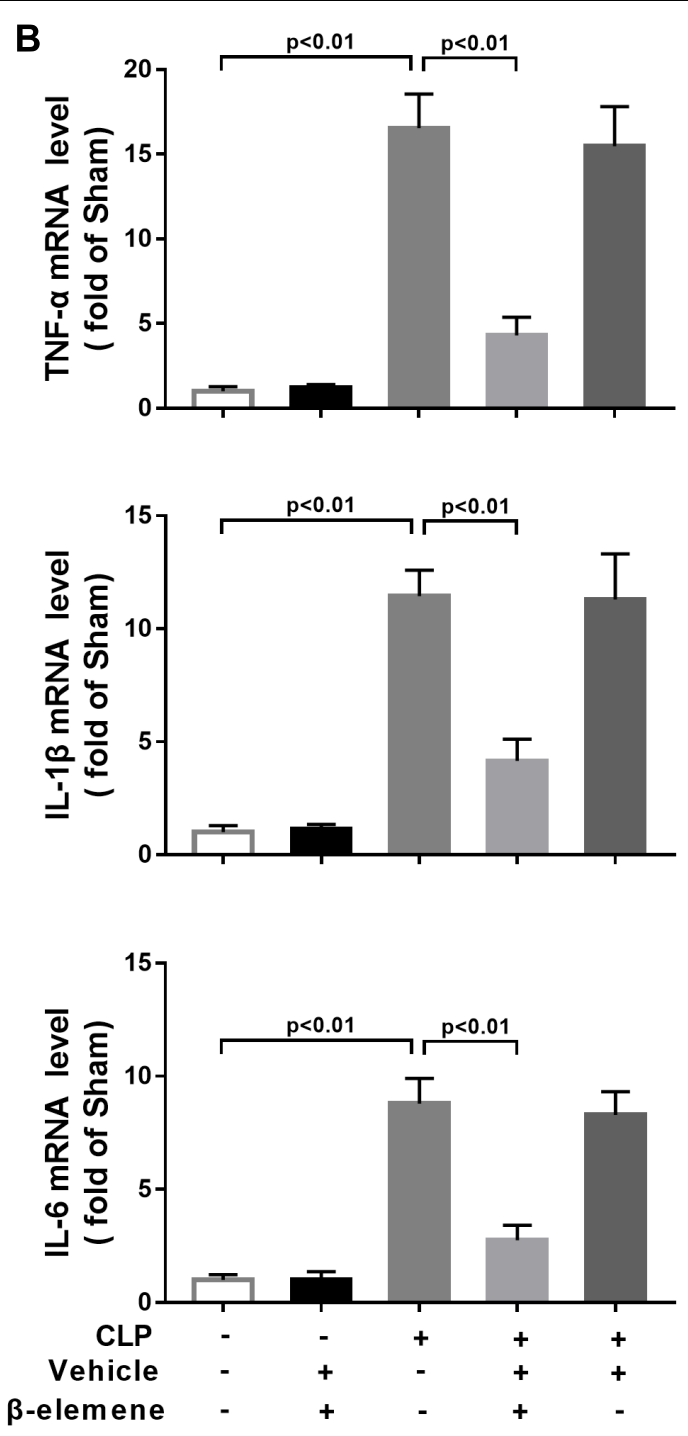

FIGURE 8 | $\beta$-Elemene reduced p38 MAPK phosphorylation and expressions of pro-inflammatory cytokines in the hippocampus of CLP mice. (A) Western blotting detected that $\beta$-elemene significantly weakened the phosphorylation of p38 $[\mathrm{F}(4,15)=44.95]$ and p65 $[\mathrm{F}(4,15)=58.7]$ normalized to that of $\mathrm{GAPDH}$. (B) RT-PCR detected that $\beta$-elemene significantly reduced the mRNA expressions of TNF- $\alpha[F(4,15)=83.98], I L-1 \beta[F(4,15)=64.01]$ and IL-6 [F(4, 15) $=73.18]$. GAPDH was used as a loading control. $n=4$. Two-way ANOVA revealed a significant difference at $p<0.05$.

(Figure 6B). Jackson et al. found that the activated form of RAC1 promoted the autophosphorylation and substrate phosphorylation of MLK3, as an upstream activator of the p38 pathway (Jackson et al., 2016). In this study, $\beta$-elemene reduced MLK3 and p38 phosphorylation in SAE mice (Figures 5, 6A). Notably, injectable emulsion of $\beta$-elemene has been developed and approved by the CFDA for clinical cancer therapy (Zhai et al., 2018). Therefore, it is a novel choice for SAE treatment.

However, this study still has some limitations. First, the possibility that $\beta$-elemene inhibited neuroinflammation by affecting intraabdominal inflammation cannot be ruled out, because it was intraperitoneally administered. Given that elemene can pass through the blood-brain barrier
(Wu et al., 2009), $\beta$-elemene may directly suppress microgliamediated neuroinflammation, playing a major role in relieving the cognitive impairment of CLP mice. Second, this study was only preliminarily explored the relationship between $\beta$-elemene and RAC1, so the regulatory mechanisms still need further indepth studies. In summary, $\beta$-elemene alleviated SAE in mice by suppressing the RAC1/MLK3/p38 signaling pathway. Therefore, $\beta$-elemene may be a potential candidate for treating SAE.

\section{ETHICS STATEMENT}

All subjects gave written informed consent in accordance with the Declaration of Helsinki. The protocol was 
approved by the Nanjing Medical University Animal Care and Use Committee.

\section{AUTHOR CONTRIBUTIONS}

CP designed the experiments, performed the experiments, analyzed the results, and drafted the manuscript. YS and QM carried out the behavioral measure. LJ carried out the cell cultures. LC and YZ carried out the western blotting analysis, immunohistochemical staining, and RTPCR analysis. HB conceived of the study, participated in its design and coordination, and helped to draft

\section{REFERENCES}

Barichello, T., Martins, M. R., Reinke, A., Feier, G., Ritter, C., Quevedo, J., et al. (2005). Cognitive impairment in sepsis survivors from cecal ligation and perforation. Crit. Care Med. 33, 221-223; discussion 262-263. doi: 10.1097/01. CCM.0000150741.12906.BD

Bid, H. K., Roberts, R. D., Manchanda, P. K., and Houghton, P. J. (2013). RAC1: an emerging therapeutic option for targeting cancer angiogenesis and metastasis. Mol. Cancer Ther. 12, 1925-1934. doi: 10.1158/1535-7163.MCT-13-0164

Chaudhry, N., and Duggal, A. K. (2014). Sepsis associated encephalopathy. $A d v$. Med. 2014:762320. doi: 10.1155/2014/762320

Chavan, S. S., Huerta, P. T., Robbiati, S., Valdes-Ferrer, S. I., Ochani, M., Dancho, M., et al. (2012). HMGB1 mediates cognitive impairment in sepsis survivors. Mol. Med. 18, 930-937. doi: 10.2119/molmed.2012.00195

Chen, Q. Y., Xu, L. Q., Jiao, D. M., Yao, Q. H., Wang, Y. Y., Hu, H. Z., et al. (2011). Silencing of RAC1 modifies lung cancer cell migration, invasion and actin cytoskeleton rearrangements and enhances chemosensitivity to antitumor drugs. Int. J. Mol. Med. 28, 769-776. doi: 10.3892/ijmm.2011.775

Cuenda, A., and Rousseau, S. (2007). p38 MAP-kinases pathway regulation, function and role in human diseases. Biochim. Biophys. Acta 1773, 1358-1375. doi: 10.1016/j.bbamcr.2007.03.010

D'Ambrosi, N., Rossi, S., Gerbino, V., and Cozzolino, M. (2014). RAC1 at the crossroad of actin dynamics and neuroinflammation in amyotrophic lateral sclerosis. Front. Cell. Neurosci. 8:279. doi: 10.3389/fncel.2014.00279

Fang, Y., Kang, Y., Zou, H., Cheng, X., Xie, T., Shi, L., et al. (2018). beta-elemene attenuates macrophage activation and proinflammatory factor production via crosstalk with Wnt/beta-catenin signaling pathway. Fitoterapia 124, 92-102. doi: 10.1016/j.fitote.2017.10.015

Gallo, K. A., and Johnson, G. L. (2002). Mixed-lineage kinase control of JNK and p38 MAPK pathways. Nat. Rev. Mol. Cell Biol. 3, 663-672. doi: 10.1038/nrm906

Henn, A., Lund, S., Hedtjarn, M., Schrattenholz, A., Porzgen, P., and Leist, M. (2009). The suitability of BV2 cells as alternative model system for primary microglia cultures or for animal experiments examining brain inflammation. Altex 26, 83-94. doi: 10.14573/altex.2009.2.83

Hernandez, E., De La Mota-Peynado, A., Dharmawardhane, S., and Vlaar, C. P. (2010). Novel inhibitors of RAC1 in metastatic breast cancer. P. R. Health Sci. J. 29, 348-356.

Hwaiz, R., Hasan, Z., Rahman, M., Zhang, S., Palani, K., Syk, I., et al. (2013). RAC1 signaling regulates sepsis-induced pathologic inflammation in the lung via attenuation of Mac-1 expression and CXC chemokine formation. J. Surg. Res. 183, 798-807. doi: 10.1016/j.jss.2013.02.045

Imamura, Y., Wang, H., Matsumoto, N., Muroya, T., Shimazaki, J., Ogura, H., et al. (2011). Interleukin-1beta causes long-term potentiation deficiency in a mouse model of septic encephalopathy. Neuroscience 187, 63-69. doi: 10.1016/ j.neuroscience.2011.04.063

Jackson, J. W., Singh, M. V., Singh, V. B., Jones, L. D., Davidson, G. A., Ture, S., et al. (2016). Novel antiplatelet activity of minocycline involves inhibition of MLK3-p38 mitogen activated protein kinase axis. PLoS One 11:e0157115. doi: 10.1371/journal.pone.0157115 the manuscript. All the authors read and approved the final manuscript.

\section{FUNDING}

This work were supported by the Jiangsu Postdoctoral Research Funding Scheme (grant number 2018K241C), the National Natural Science Foundation of China (grant number 81401620), Nanjing Medical Science and Technology Development Foundation (grant number QRX17019), and Nanjing Science and Technology Development Foundation (grant number 201715033).

Jacob, A., Brorson, J. R., and Alexander, J. J. (2011). Septic encephalopathy: inflammation in man and mouse. Neurochem. Int. 58, 472-476. doi: 10.1016/ j.neuint.2011.01.004

Kelly, A., Vereker, E., Nolan, Y., Brady, M., Barry, C., Loscher, C. E., et al. (2003). Activation of p38 plays a pivotal role in the inhibitory effect of lipopolysaccharide and interleukin-1 beta on long term potentiation in rat dentate gyrus. J. Biol. Chem. 278, 19453-19462. doi: 10.1074/jbc.M301938200

Kuijk, L. M., Beekman, J. M., Koster, J., Waterham, H. R., Frenkel, J., and Coffer, P. J. (2008). HMG-CoA reductase inhibition induces IL-1beta release through RAC1/PI3K/PKB-dependent caspase-1 activation. Blood 112, 3563-3573. doi: 10.1182/blood-2008-03-144667

Kwon, T., Kwon, D. Y., Chun, J., Kim, J. H., and Kang, S. S. (2000). Akt protein kinase inhibits RAC1-GTP binding through phosphorylation at serine 71 of RAC1. J. Biol. Chem. 275, 423-428. doi: 10.1074/jbc.275.1.423

Li, Q. Q., Wang, G., Huang, F., Banda, M., and Reed, E. (2010). Antineoplastic effect of beta-elemene on prostate cancer cells and other types of solid tumour cells. J. Pharm. Pharmacol. 62, 1018-1027. doi: 10.1111/j.2042-7158.2010.01135.x

Liu, D., Ye, Y., Xu, L., Yuan, W., and Zhang, Q. (2018). Icariin and mesenchymal stem cells synergistically promote angiogenesis and neurogenesis after cerebral ischemia via PI3K and ERK1/2 pathways. Biomed. Pharmacother. 108, 663-669. doi: 10.1016/j.biopha.2018.09.071

Liu, M., Mao, L., Daoud, A., Hassan, W., Zhou, L., Lin, J., et al. (2015). betaelemene inhibits monocyte-endothelial cells interactions via reactive oxygen species/MAPK/NF-kappaB signaling pathway in vitro. Eur. J. Pharmacol. 766, 37-45. doi: 10.1016/j.ejphar.2015.09.032

Lobo, R., Prabhu, K. S., Shirwaikar, A., and Shirwaikar, A. (2009). Curcuma zedoaria Rosc. (white turmeric): a review of its chemical, pharmacological and ethnomedicinal properties. J. Pharm. Pharmacol. 61, 13-21. doi: 10.1211/jpp/ 61.01 .0003

Michels, M., Danielski, L. G., Dal-Pizzol, F., and Petronilho, F. (2014). Neuroinflammation: microglial activation during sepsis. Curr. Neurovasc. Res. 11, 262-270. doi: 10.2174/1567202611666140520122744

Piva, S., McCreadie, V. A., and Latronico, N. (2015). Neuroinflammation in sepsis: sepsis associated delirium. Cardiovasc. Hematol. Disord. Drug Targets 15, 10-18. doi: 10.2174/1871529X15666150108112452

Qin, C., Liu, R., and Liu, H. (2015). The conflicting role of RAC1 in inflammation. Inflamm. Cell Signal. 2, 1-14. doi: 10.14800/ics.922

Riento, K., Totty, N., Villalonga, P., Garg, R., Guasch, R., and Ridley, A. J. (2005). RhoE function is regulated by ROCK I-mediated phosphorylation. EMBO J. 24, 1170-1180. doi: 10.1038/sj.emboj.7600612

Sanlioglu, S., Williams, C. M., Samavati, L., Butler, N. S., Wang, G., McCray, P. B. Jr., et al. (2001). Lipopolysaccharide induces RAC1-dependent reactive oxygen species formation and coordinates tumor necrosis factor-alpha secretion through IKK regulation of NF-kappa B. J. Biol. Chem. 276, 30188-30198. doi: 10.1074/jbc.M102061200

Schoentaube, J., Olling, A., Tatge, H., Just, I., and Gerhard, R. (2009). Serine71 phosphorylation of RAC1/Cdc42 diminishes the pathogenic effect of clostridium difficile toxin A. Cell Microbiol. 11, 1816-1826. doi: 10.1111/j.14625822.2009.01373.x 
Schwalm, M. T., Pasquali, M., Miguel, S. P., Dos Santos, J. P., Vuolo, F., Comim, C. M., et al. (2014). Acute brain inflammation and oxidative damage are related to long-term cognitive deficits and markers of neurodegeneration in sepsis-survivor rats. Mol. Neurobiol. 49, 380-385. doi: 10.1007/s12035-0138526-3

Schwarz, J., Proff, J., Havemeier, A., Ladwein, M., Rottner, K., Barlag, B., et al. (2012). Serine-71 phosphorylation of RAC1 modulates downstream signaling. PLoS One 7:e44358. doi: 10.1371/journal.pone.0044358

Semmler, A., Frisch, C., Debeir, T., Ramanathan, M., Okulla, T., Klockgether, T., et al. (2007). Long-term cognitive impairment, neuronal loss and reduced cortical cholinergic innervation after recovery from sepsis in a rodent model. Exp. Neurol. 204, 733-740. doi: 10.1016/j.expneurol.2007.01.003

Semmler, A., Hermann, S., Mormann, F., Weberpals, M., Paxian, S. A., Okulla, T., et al. (2008). Sepsis causes neuroinflammation and concomitant decrease of cerebral metabolism. J. Neuroinflamm. 5:38. doi: 10.1186/1742-2094-5-38

Sonneville, R., de Montmollin, E., Poujade, J., Garrouste-Orgeas, M., Souweine, B., Darmon, M., et al. (2017). Potentially modifiable factors contributing to sepsisassociated encephalopathy. Intens. Care Med. 43, 1075-1084. doi: 10.1007/ s00134-017-4807-z

Wiltgen, B. J., Sanders, M. J., Ferguson, C., Homanics, G. E., and Fanselow, M. S. (2005). Trace fear conditioning is enhanced in mice lacking the delta subunit of the GABAA receptor. Learn. Mem. 12, 327-333. doi: 10.1101/lm.89705

Wu, X. S., Xie, T., Lin, J., Fan, H. Z., Huang-Fu, H. J., Ni, L. F., et al. (2009). An investigation of the ability of elemene to pass through the blood-brain barrier and its effect on brain carcinomas. J. Pharm. Pharmacol. 61, 1653-1656. doi: $10.1211 /$ jpp/61.12.0010

Yao, H. Y., Chen, L., Xu, C., Wang, J., Chen, J., Xie, Q. M., et al. (2011). Inhibition of Rac activity alleviates lipopolysaccharide-induced acute pulmonary injury in mice. Biochim. Biophys. Acta 1810, 666-674. doi: 10.1016/j.bbagen.2011. 03.020

Yao, Y. Q., Ding, X., Jia, Y. C., Huang, C. X., Wang, Y. Z., and Xu, Y. H. (2008). Anti-tumor effect of beta-elemene in glioblastoma cells depends on p38 MAPK activation. Cancer Lett. 264, 127-134. doi: 10.1016/j.canlet.2008.01.049
Yoshida, T., Zhang, Y., Rivera Rosado, L. A., Chen, J., Khan, T., Moon, S. Y., et al. (2010). Blockade of RAC1 activity induces G1 cell cycle arrest or apoptosis in breast cancer cells through downregulation of cyclin D1, survivin, and X-linked inhibitor of apoptosis protein. Mol. Cancer Ther. 9, 1657-1668. doi: 10.1158/ 1535-7163.MCT-09-0906

Zhai, B., Zeng, Y., Zeng, Z., Zhang, N., Li, C., Zeng, Y., et al. (2018). Drug delivery systems for elemene, its main active ingredient beta-elemene, and its derivatives in cancer therapy. Int. J. Nanomed. 13, 6279-6296. doi: 10.2147/IJN.S174527

Zhang, Q. G., Wang, X. T., Han, D., Yin, X. H., Zhang, G. Y., and Xu, T. L. (2006). Akt inhibits MLK3/JNK3 signaling by inactivating RAC1: a protective mechanism against ischemic brain injury. J. Neurochem. 98, 1886-1898. doi: 10.1111/j.1471-4159.2006.04020.x

Zhang, R., Tian, A., Shi, X., Yu, H., and Chen, L. (2010). Downregulation of IL-17 and IFN-gamma in the optic nerve by beta-elemene in experimental autoimmune encephalomyelitis. Int. Immunopharmacol. 10, 738-743. doi: 10. 1016/j.intimp.2010.04.003

Zhang, T., Lu, X., Beier, F., and Feng, Q. (2011). RAC1 activation induces tumour necrosis factor-alpha expression and cardiac dysfunction in endotoxemia. J. Cell Mol. Med. 15, 1109-1121. doi: 10.1111/j.1582-4934.2010.01095.x

Zuo, Y., Shields, S. K., and Chakraborty, C. (2006). Enhanced intrinsic migration of aggressive breast cancer cells by inhibition of RAC1 GTPase. Biochem. Biophys. Res. Commun. 351, 361-367. doi: 10.1016/j.bbrc.2006.10.043

Conflict of Interest Statement: The authors declare that the research was conducted in the absence of any commercial or financial relationships that could be construed as a potential conflict of interest.

Copyright (c) 2019 Pan, Si, Meng, Jing, Chen, Zhang and Bao. This is an open-access article distributed under the terms of the Creative Commons Attribution License (CC BY). The use, distribution or reproduction in other forums is permitted, provided the original author(s) and the copyright owner(s) are credited and that the original publication in this journal is cited, in accordance with accepted academic practice. No use, distribution or reproduction is permitted which does not comply with these terms. 